\title{
20(s)-ginseonside-Rg3 modulation of AMPK/FoxO3 signaling to attenuate mitochondrial dysfunction in a dexamethasone-injured C2C12 myotube-based model of skeletal atrophy in vitro
}

\author{
MANYING WANG ${ }^{1,2^{*}}$, RUI JIANG $^{1 *}$, JIANZENG LIU $^{2}$, XIAOHAO XU ${ }^{1}$, \\ GUANG SUN $^{1}$, DAQING ZHAO ${ }^{2,3}$ and LIWEI SUN ${ }^{1,3}$
${ }^{1}$ Research Center of Traditional Chinese Medicine, The Affiliated Hospital to Changchun University of Chinese Medicine, Changchun, Jilin 130021; ${ }^{2}$ Jilin Ginseng Academy, Changchun University of Chinese Medicine;
${ }^{3}$ Key Laboratory of Active Substances and Biological Mechanisms of Ginseng Efficacy, Ministry of Education, Changchun, Jilin 130117, P.R. China

Received July 31, 2020; Accepted January 18, 2021

DOI: $10.3892 / \mathrm{mmr} .2021 .11945$

\begin{abstract}
Muscle atrophy, a side effect from administration of the anti-inflammatory medication dexamethasone (DEX), is preventable by concomitant administration of the major monomeric constituent of Panax ginseng C.A. Meyer, 20(S)-ginsenoside Rg3 (S-Rg3). Putative S-Rg3-associated prevention of DEX-induced muscle atrophy may involve $\mathrm{S}-\mathrm{Rg} 3$ mitigation of DEX-induced mitochondrial dysfunction. In the present study, MTT assays revealed enhanced cell viability following S-Rg3 treatment of DEX-injured C2C12 myotubes. Subsequent PCR and western blotting results demonstrated S-Rg3-induced reduction of expression of muscle atrophy F-box protein (atrogin-1) and muscle RING-finger protein-1, proteins previously linked to muscle atrophy. Additionally, S-Rg3 treatment of DEX-injured myotubes led to aggregation of $\operatorname{Rg} 3$ monomers in cells and dose-dependent increases in cellular mitochondrial basal respiratory oxygen consumption rate and intracellular ATP levels compared with their levels in untreated DEX-injured myotubes. In addition, S-Rg3 treatment significantly reversed DEX-induced reductions of expression
\end{abstract}

Correspondence to: Professor Liwei Sun, Research Center of Traditional Chinese Medicine, The Affiliated Hospital to Changchun University of Chinese Medicine, 1478 Gongnong Street, Changchun, Jilin 130021, P.R. China

E-mail: sunnylilwei@163.com

Professor Daqing Zhao, Jilin Ginseng Academy, Changchun University of Chinese Medicine, 1035 Boshuo Road, Changchun, Jilin 130117, P.R. China

E-mail: zhaodaqing1963@163.com

*Contributed equally

Key words: 20(S)-ginsenoside Rg3, mitochondrial dysfunction, skeletal muscle atrophy, AMP-activated protein kinase/forkhead box $\mathrm{O} 3$ of key mitochondrial respiratory electron transport chain subunits of protein complexes II, III and V in DEX-injured myotube cells. Furthermore, S-Rg3 alleviation of mitochondrial dysfunction associated with DEX-induced injury of $\mathrm{C} 2 \mathrm{C} 12$ myotubes was linked to $\mathrm{S}-\mathrm{Rg} 3$-associated decreases in both forkhead box $\mathrm{O} 3$ (FoxO3) protein expression and phosphorylation of AMP-activated protein kinase (AMPK). Collectively, these results implicate S-Rg3 modulation of signaling within the AMPK-FoxO3 pathway as a putative mechanism underlying S-Rg3 alleviation of DEX-induced muscle atrophy.

\section{Introduction}

In clinical settings, the synthetic glucocorticoid (GC) dexamethasone (DEX) and its derivatives are routinely used to treat inflammatory disorders (1). However, excessive use of DEX has been linked to negative human health effects that include glucose metabolic dysfunction, insulin resistance, mitochondrial dysfunction and muscle atrophy $(2,3)$. Muscle atrophy may be linked to long-term DEX treatment, which leads to altered mitochondrial morphology, aggregation (4), enlargement and compromised mitochondrial oxidative capacity (5). Due to the fact that muscle atrophy can greatly affect the well-being of patients (6), alleviation of DEX-induced muscle atrophy would be of great benefit to patients with inflammatory diseases who rely on DEX treatment.

Chronic corticosteroid treatment has been shown in numerous clinical and animal studies to cause DNA oxidative damage resulting from disruption of mitochondrial morphology and oxidative capacity (7). Indeed, such effects have been reported frequently in conjunction with clinically apparent mitochondrial dysfunction and skeletal muscle atrophy linked to long-term and/or high-dose GC treatments $(8,9)$. Muscle atrophy associated with DEX treatment has been experimentally linked to decreased protein synthesis accompanied by increased protein degradation (10), both of which appear to be triggered by ubiquitin-proteasome system activation involving muscle atrophy F-box protein (atrogin-1) and muscle RING 
finger-1 (MuRF1) protein (11). These two muscle-specific proteins function as E3 ubiquitin ligases that are expressed early during the muscle deterioration process before muscle loss becomes apparent and thus may participate as key participants in DEX-associated muscle atrophy.

Notably, GCs induction of muscle atrophy-associated protein breakdown appears to involve effects on transcription factor production, specifically of forkhead box (Fox) O1, FoxO3a (12) and GSK3 $\beta$ (13), that trigger ubiquitin proteasome system-dependent proteolysis of muscle proteins and subsequent autophagy (14). Meanwhile, studies of muscle atrophy induced by denervation have identified increased mitochondrial fission and resulting fragmentation as key signals responsible for triggering the AMP-activated kinaseforkhead box O3 (AMPK-FoxO3) signaling pathway $(15,16)$. Mitochondria are organelles that provide energy to cells. Matching energy supply with energy demand is coordinated through various processes and is critical for cellular adaptation and survival under changing conditions (17). Damage to mitochondrial function can lead to insufficient ATP supply and activation of the AMPK/FoxO3 pathway to cause muscle atrophy. After AMPK-FoxO3 pathway activation is triggered via the metabolic sensor AMPK, FoxO3 is dephosphorylated then undergoes nuclear translocation. Once in the nucleus, it acts as a transcription factor that upregulates atrophy-inducing genes expression that engage in events culminating in protein degradation and eventual atrophy of skeletal muscle (18). In support of this mechanism, findings of a previous in vitro study suggested that nuclear translocation of FoxO3 led to upregulation of MuRF1 expression and subsequent myotube atrophy (19).

Numerous studies have shed light on several putative mechanisms that may be responsible for observed beneficial effects of Panax ginseng compounds on muscle health. For example, studies in rats have shown that treatment with traditional Chinese herbal medicine Panax ginseng prevented muscle atrophy (20), while a diabetic mouse study demonstrated that panaxatriols extracted from ginseng exerted a similar effect on skeletal muscle (21). In one study, treatment with the panaxatriol Rg1, a ginsenoside, prevented degradation of $\mathrm{C} 2 \mathrm{C} 12$ myotube muscle protein through regulation of the AKT/mTOR/FoxO signaling pathway (22), with prevention of atrophy attributed in another study to AKT/mTOR pathway activation (23). Another panaxatriol, 20(s)-ginsenoside Rg3 (S-Rg3), a major monomeric ginsenoside also isolated from Panax ginseng, appeared to prevent TNF- $\alpha$-induced myotube atrophy (24). Our previous study demonstrated that S-Rg3 could prevent myotube atrophy by promoting myoblast differentiation (25). However, no investigations of mechanisms involved in $\mathrm{S}-\mathrm{Rg} 3$ prevention of myotube atrophy have focused on S-Rg3 effects on mitochondrial function, even though DEX is known to cause mitochondrial dysfunction. Therefore, the present study investigated whether S-Rg3 treatment of DEX-injured C2C12 myotubes could prevent muscle atrophy by alleviating mitochondrial dysfunction induced by DEX.

\section{Materials and methods}

Materials. Chemicals, reagents and kits were obtained from commercial sources as follows: S-Rg3 (Urchem Sinopharm
Chemical Reagent Co., Ltd.), dexamethasone (DEX) and 3-(4,5-dimethylthiazol-2-yl)-2,5-diphenyltetrazolium bromide (MTT) (Sigma-Aldrich; Merck KGaA), mouse skeletal muscle-derived C2C12 myoblasts (American Type Culture Collection), fetal bovine serum (FBS; Clark BioScience), horse serum (Gibco; Thermo Fisher Scientific, Inc.), antibodies against cleaved caspase-3 (cat. no. 9661), phosphorylated-AMPK (p-AMPK; cat. no. 2535), AMPK (cat. no. 2532), Bcl-2 (cat. no. 3498), Bax (cat. no. 2772), FoxO3 (cat. no. 2497), histone H3 (cat. no. 4499) and $\beta$-tubulin (cat. no. 2146) were obtained from Cell Signaling Technology, Inc.; atrogin-1 antibody (cat. no. ab168372) and total OXPHOS Rodent WB Antibody Cocktail (anti-complex I, II, III, IV, V; cat. no. ab110413) were obtained from Abcam; antibody specific for muscle-specific RING finger 1 (MuRF1; cat. no. bs-2539R) was purchased from BIOSS. Chemiluminescence reagents was purchased from Santa Cruz Biotechnology.

Cell culture and induction of cell differentiation. $\mathrm{C} 2 \mathrm{C} 12$ myoblasts were cultivated via incubation at $37^{\circ} \mathrm{C}$ in an atmosphere containing $5 \% \mathrm{CO}_{2}$ with humidification in medium containing Dulbecco's modified Eagle's medium (DMEM) with $25 \mathrm{mM}$ glucose, $10 \%$ FBS, $100 \mu \mathrm{g} / \mathrm{ml}$ streptomycin and 100 units/ml penicillin until $70-80 \%$ confluence. Next, myoblasts were seeded at $7.5 \times 10^{4}$ cells/well in 6 -well plates or at $5 \times 10^{3}$ cells/well in 96-well plates. Myoblast fusion to form C2C12 myotubes was induced by culturing cells for 5 days in DMEM containing 2\% horse serum and $25 \mathrm{mM}$ glucose (26).

MTT cell viability assay. MTT assays were conducted to monitor cell viability $(27,28)$ as follows: C2C12 myotubes treated for 24 to $48 \mathrm{~h}$ with various concentrations of $\operatorname{DEX}(0$, $25,50,100$ or $200 \mu \mathrm{M})(29)$ or with $\mathrm{S}-\operatorname{Rg} 3(0,0.02,0.2$ or $2 \mu \mathrm{M})$ and/or DEX $(200 \mu \mathrm{M})$ for $24 \mathrm{~h}$ were incubated with MTT $(0.5 \mathrm{mg} / \mathrm{ml})$ for $4 \mathrm{~h}$. All treatments were conducted at $37^{\circ} \mathrm{C}$. Next, formazan dissolved in $150 \mu \mathrm{l}$ DMSO was added to wells then measurements of absorbance levels of wells were taken at a wavelength of $490 \mathrm{~nm}$ at room temperature. Calculations of cell viability were performed that generated results as percentages of viable cells relative to vehicle control.

Intracellular ATP level measurement. After C2C12 myotubes were treated with various concentrations of $\operatorname{DEX}(0,25$, 50,100 or $200 \mu \mathrm{M}$ ) for $24 \mathrm{~h}, 50 \mu \mathrm{M}$ DEX for $0,3,6,12$ or $24 \mathrm{~h}$ or $\mathrm{S}-\operatorname{Rg} 3(0,0.02,0.2$ or $2 \mu \mathrm{M})$ and/or $\operatorname{DEX}(50 \mu \mathrm{M})$ for $6 \mathrm{~h}$, all at $37^{\circ} \mathrm{C}, \mathrm{C} 2 \mathrm{C} 12$ myotubes were lysed using ATP lysis buffer composed of $0.5 \%$ Triton X-100, $100 \mathrm{mM}$ glycine, $\mathrm{pH} 7.4$ then lysates were centrifuged at $15,000 \mathrm{x}$ g for $10 \mathrm{~min}$ at $4^{\circ} \mathrm{C}(30)$. Supernatants were collected and an ATP Bioluminescent Assay kit (Promega Corporation) was used to measure intracellular ATP levels in supernatants on ice according to the manufacturer's instructions.

Oxygen consumption rate $(O C R)$ measurement. Basal respiration rates of mitochondria were measured via previously reported methods using a kit (MitoXpress ${ }^{\circledR}$ Xtra Oxygen Consumption Assay; Agilent Technologies, Inc.) (31). In brief, C2C12 myoblasts previously seeded into 96-well microplates (Corning, Inc.) were incubated for 5 day to allow them to differentiate. $\mathrm{C} 2 \mathrm{C} 12$ myotubes were then treated with various 
concentrations of $\operatorname{DEX}(0,25,50,100$ or $200 \mu \mathrm{M})$ for $24 \mathrm{~h}$, $50 \mu \mathrm{M}$ DEX for $0,3,6,12$ or $24 \mathrm{~h}$ or $\mathrm{S}-\operatorname{Rg} 3(0,0.02,0.2$ or $2 \mu \mathrm{M})$ and/or DEX $(50 \mu \mathrm{M})$ for $6 \mathrm{~h}$, all at $37^{\circ} \mathrm{C}$. OCRs were measured using a plate reader at room temperature (Cytation 5; BioTek Instruments, Inc.).

Transmission electron microscopy. C2C12 myotubes were treated with $50 \mu \mathrm{M} \mathrm{DEX}$ with or without $0.2 \mu \mathrm{M} \mathrm{S}-\mathrm{Rg} 3$ for $6 \mathrm{~h}$ at $37^{\circ} \mathrm{C}$. After cells were fixed by 2 -h immersion in $2.5 \%$ glutaraldehyde fixative at $4^{\circ} \mathrm{C}$, they were post-fixed in $1 \% \mathrm{OsO}_{4}$ at room temperature for $2 \mathrm{~h}$, then dehydrated in an ascending alcohol series and embedded in Epon resin. Using a microtome (Leica UC7; Leica Microsystems, Inc.), ultrathin sections (60-80 nm) were sliced from embedded cell blocks, then sections were stained with $2 \%$ uranyl acetate and lead citrate at room temperature for $15 \mathrm{~min}$ each, then examined using an electron microscope (Tecnai G2 20 TWIN, FEI; Thermo Fisher Scientific, Inc.). To measure DEX-induced changes in mitochondrial number and area, images were processed using Image-Pro Plus 6.0 software (Media Cybernetics, Inc.) and data were analyzed by a statistician who had no access to the images (blind data analysis).

Mitochondrial membrane potential detection. $\mathrm{C} 2 \mathrm{C} 12$ myotubes were treated with $50 \mu \mathrm{M}$ DEX and $0,0.02$, 0.2 or $2 \mu \mathrm{M} \mathrm{S}-\mathrm{Rg} 3$ for $6 \mathrm{~h}$ at $37^{\circ} \mathrm{C}$. A JC-1 fluorescent probe (Beyotime Institute of Biotechnology) was used to estimate the effect of S-Rg3 on mitochondrial membrane potential using the manufacturer's instructions provided with the probe, but with modifications (32). After 5-days culture of C2C12 myoblasts seeded at $7.5 \times 10^{4}$ cells/well in 6 -well plates, cells were treated for $6 \mathrm{~h}$ with $\mathrm{S}-\mathrm{Rg} 3$ and DEX, each at various concentrations. Cells were next incubated in the dark with JC-1 stain for $20 \mathrm{~min}$ at $37^{\circ} \mathrm{C}$ then stained cells were immediately analyzed using flow cytometry (FACScan; BD Biosciences).

$R N A$ extraction and reverse transcription-quantitative $(R T-q)$ $P C R$. C2C12 myoblasts seeded at a density of $7.5 \times 10^{4}$ cells/well into 6-well plates were induced with DMEM containing $2 \%$ horse serum and $25 \mathrm{mM}$ glucose for 5 days, then treated with $200 \mu \mathrm{M}$ DEX for $24 \mathrm{~h}$ and collected on ice. After extraction of total RNA using TRIzol ${ }^{\circledR}$ reagent (Invitrogen; Thermo Fisher Scientific, Inc.) according to the manufacturer's protocols, cDNA was generated from total RNA $(1 \mu \mathrm{g})$ via a reverse transcription kit (cat. no. KR118-02; Tiangen Biotech Co., Ltd.) on ice, then a real-time PCR system (Eppendorf) was used to conduct qPCR. The following thermocycling conditions were used for the qPCR: Initial denaturation at $95^{\circ} \mathrm{C}$ for $5 \mathrm{~min}$; followed by 40 cycles of $95^{\circ} \mathrm{C}$ for $15 \mathrm{sec}, 60^{\circ} \mathrm{C}$ for $30 \mathrm{sec}$ and $72^{\circ} \mathrm{C}$ for $30 \mathrm{sec}$. Labeling of amplification products generated via qPCR was achieved using SYBR Green Master Mix (Takara Biotechnology Co., Ltd.) and gene-specific primers. Primer sequences were as follows: MuRF1, 5'-TGG AAACGCTATGGAGAACC-3' (forward) and 5'-ATTCGC AGCCTGGAAGATG-3' (reverse); Atrogin-1, 5'-CTGGCA GCAGCAGCTGAATAG-3' (forward) and 5'-CACATGCAG GTCTGGGGCTGC-3' (reverse); actin, 5'-AGGCCCAGA GCAAGAGAGGTA-3' (forward) and 5'-CCATGTCGTCCC AGTTGGTAA-3' (reverse). Amplification products generated from housekeeping gene actin mRNA were used to normalize the levels of the other products. Relative mRNA expression was calculated based on the $2^{-\Delta \Delta C q}$ method (33).

Western blot analysis. C2C12 myotubes were treated with 50 or $200 \mu \mathrm{M}$ DEX and $0,0.02,0.2$ or $2 \mu \mathrm{M} \mathrm{S}-\mathrm{Rg} 3$ for 6 or $24 \mathrm{~h}$ at $37^{\circ} \mathrm{C}$. After washing cells twice with phosphate-buffered saline, they were suspended in RIPA lysis buffer (Beyotime Institute of Biotechnology) and allowed to lyse for $30 \mathrm{~min}$ at $4^{\circ} \mathrm{C}$. Nuclear and cytoplasmic proteins were isolated separately using a BestBio BB-3102 kit (BestBio). Protein concentration was determined using a BCA Protein Assay kit (Beyotime Institute of Biotechnology). Lysates $(30 \mu \mathrm{g}$ total/lane) were separated via $12 \%$ SDS-PAGE followed by electro-transfer to polyvinylidene fluoride (PVDF) membranes. After blocking of membranes in 5\% bovine serum albumin (cat. no. 4240; BioFroxx) at room temperature for $1 \mathrm{~h}$, membranes were incubated overnight with 1:1,000 dilution of primary antibody at $4{ }^{\circ} \mathrm{C}$ followed by two washes and a 1-h incubation with 1:5,000 of horseradish peroxidase-conjugated goat anti-rabbit IgG (cat. no.) BA1054 and goat anti-mouse IgG (cat. no. BA1050) secondary antibodies (Boster Biological Technology) at room temperature. Proteins were visualized via chemiluminescence (ProteinSimple), then imaged using a FluorChem HD2 system (ProteinSimple). Densitometry was performed using AlphaView SA software 3.4.0.0 (ProteinSimple) (34).

Statistical analysis. All statistical analyses were performed with GraphPad Prism 7 (GraphPad Software, Inc.) and using an unpaired Student's t-test or one-way analysis of variance (ANOVA) followed by Tukey's post-hoc tests. Data are expressed as the mean \pm standard deviation of three independent experimental repeats. $\mathrm{P}<0.05$ was considered to indicate a statistically significant difference.

\section{Results}

Induction of muscle atrophy and mitochondrial dysfunction by dexamethasone (DEX) treatment. Results of experiments whereby $\mathrm{C} 2 \mathrm{C} 12$ myotubes received treatment with various doses of $\operatorname{DEX}(25,50,100$ and $200 \mu \mathrm{M})$ for 24 and $48 \mathrm{~h}$ revealed no effect on cell viability at DEX concentrations below $200 \mu \mathrm{M}$ (Fig. 1A). Meanwhile, increased levels of two muscle atrophy marker proteins, MuRF1 and atrogin-1, were observed in $\mathrm{C} 2 \mathrm{C} 12$ myotubes treated with $200 \mu \mathrm{M}$ DEX (Fig. 1B). Next, measurements of C2C12 myotube OCR and intracellular ATP level were performed to reveal the relationship between muscle atrophy and mitochondrial function. Subsequently it was observed that 24-h DEX treatment (50-200 $\mu \mathrm{M}$ DEX) led to ATP deprivation (Fig. 1C) and reduced OCR (Fig. 1D). Next, C2C12 myotubes were treated with $\operatorname{DEX}(50 \mu \mathrm{M})$ for various lengths of time, with the results revealing that 6-h DEX treatment of $\mathrm{C} 2 \mathrm{C} 12$ myotubes led to ATP deprivation (Fig. 1E) and OCR reduction (Fig. 1F). When considered together, these results demonstrated that damage to mitochondria preceded myotube atrophy, since $50 \mu \mathrm{M}$ DEX treatment of $\mathrm{C} 2 \mathrm{C} 12$ myotubes for only $6 \mathrm{~h}$ led to mitochondrial dysfunction, while 24-h treatment with $200-\mu \mathrm{M}$ DEX was necessary to induce myotube atrophy. Therefore, to generate a mitochondrial damage model the present study employed 6-h 
A

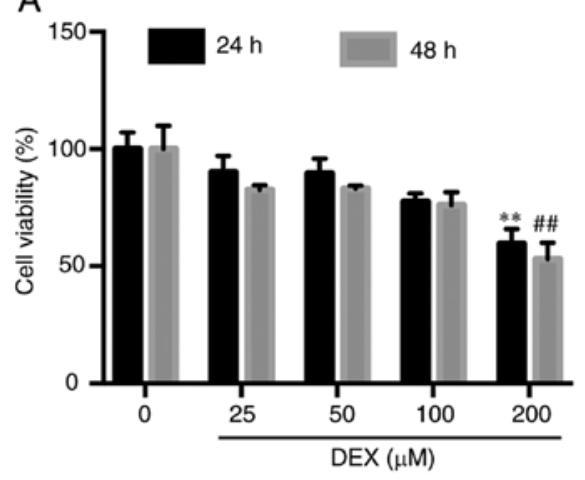

C

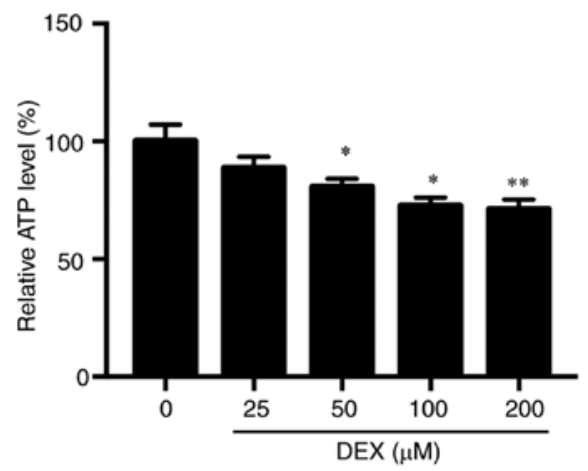

E

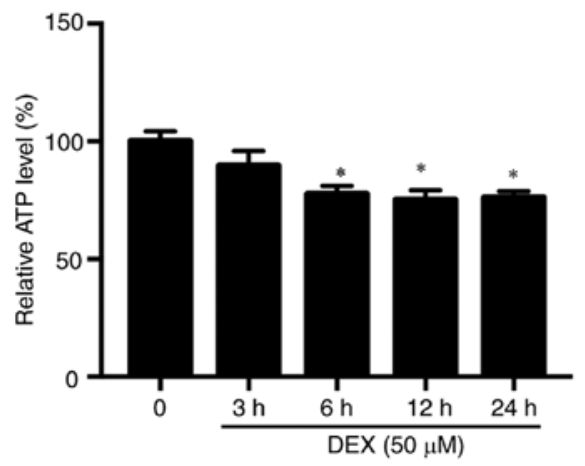

$\mathrm{B}$

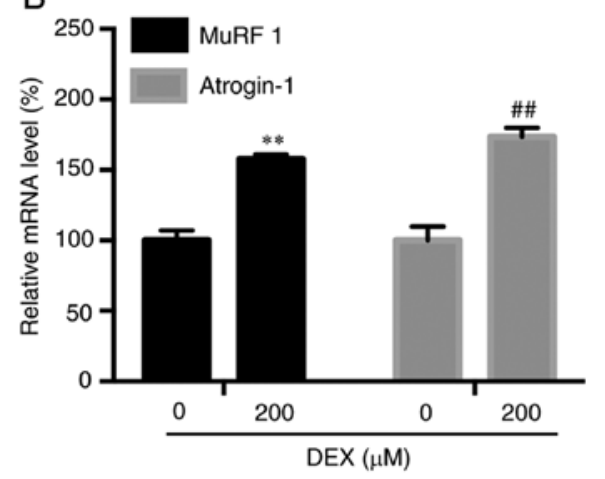

D

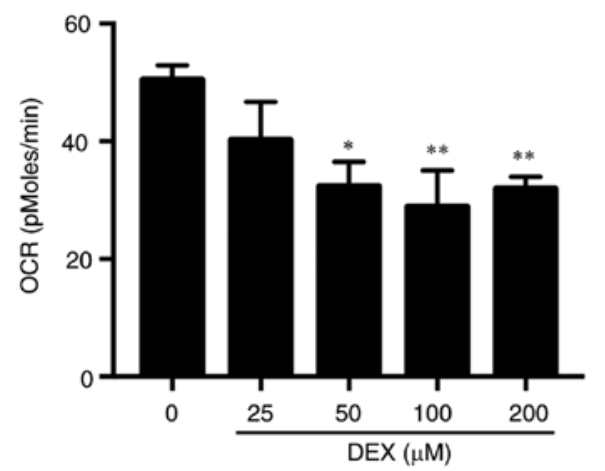

$\mathrm{F}$

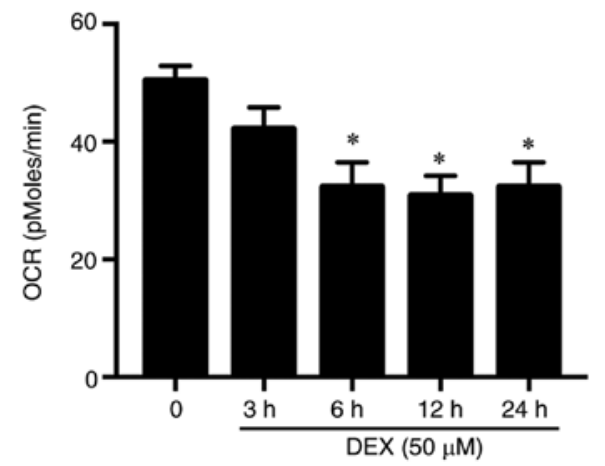

Figure 1. Muscle atrophy and mitochondrial dysfunction induced by DEX. (A) After 24 or 48 -h treatment with various doses of DEX ( $25,50,100$ and $200 \mu \mathrm{M})$, no effect on $\mathrm{C} 2 \mathrm{C} 12$ myotube viability was observed at DEX concentrations $<200 \mu \mathrm{M}$. ${ }^{* *} \mathrm{P}<0.01$ vs. CON $(24 \mathrm{~h}) ;{ }^{* \#} \mathrm{P}<0.01$ vs. CON $(48 \mathrm{~h})$. Results were analyzed via one-way ANOVA with data expressed as the mean \pm standard deviation $(\mathrm{n}=3)$. (B) DEX-treated $\mathrm{C} 2 \mathrm{C} 12$ myotube atrogen-1 and MuRF1 mRNA levels were higher compared with corresponding levels in control cells. ${ }^{* *} \mathrm{P}<0.01$ vs. CON. (MuRF1); ${ }^{\# \#} \mathrm{P}<0.01$ vs. CON (atrogin-1). Results were analyzed via Student's $\mathrm{t}$-test, with data expressed as the mean \pm standard deviation $(\mathrm{n}=3)$. (C) Intracellular ATP level was reduced following 24-h treatment with various DEX doses $(25,50,100$ and $200 \mu \mathrm{M})$. ${ }^{*} \mathrm{P}<0.05$ and ${ }^{* *} \mathrm{P}<0.01$ vs. CON. Results were analyzed via one-way ANOVA, with data expressed as the mean \pm standard deviation $(\mathrm{n}=3)$. (D) Basal respiration (OCR) measured by MitoXpress ${ }^{\circledast}$ Xtra Oxygen Consumption Assay was reduced at $24 \mathrm{~h}$ following administration of various doses of DEX $(25,50,100$ and $200 \mu \mathrm{M})$. ${ }^{*} \mathrm{P}<0.05$ and ${ }^{* *} \mathrm{P}<0.01$ vs. CON. Results were analyzed via one-way ANOVA, with data expressed as the mean \pm standard deviation ( $\mathrm{n}=3$ ). (E) Reduction of intracellular ATP level following DEX incubation for various time points in hours. "P<0.05 vs. CON. Results were analyzed via one-way ANOVA, with data expressed as the mean \pm standard deviation $(n=3)$. (F) Reduction of OCR following DEX administration for various time points in hours. " $\mathrm{P}<0.05$ vs. CON. Results were analyzed via one-way ANOVA, with data expressed as the mean \pm standard deviation $(\mathrm{n}=3)$. DEX, dexamethasone; CON, $0 \mu \mathrm{M}$ DEX; MuRF1, muscle RING finger-1; atrogin-1, muscle atrophy F-box protein; OCR, oxygen consumption rate.

administration of $50 \mu \mathrm{M}$ DEX and for the myotube atrophy model administered 24-h treatment with $200 \mu \mathrm{M}$ DEX.

Treatment with $S$ - Rg3 reverses C2C12 myotube atrophy induced by $D E X$. After verifying that DEX-induced myotube atrophy had occurred as the present study's model for skeletal muscle atrophy, effects of S-Rg3 treatment on DEX-induced injury were evaluated. DEX treatment alone decreased $\mathrm{C} 2 \mathrm{C} 12$ myotube cell viability to $68.68 \pm 1.21 \%$, while S-Rg3 treatment restored C2C12 myotube cell viability in a dose-dependent manner (Fig. 2A). The expression levels of muscle cell-specific major muscle atrophy-associated E3 ubiquitin ligases atrogin-1 and MuRF1 were examined to assess S-Rg3 effects on DEX-induced skeletal muscle cell atrophy. Notably, DEX increased expression of both atrogin-1 and MuRF1 proteins in $\mathrm{C} 2 \mathrm{C} 12$ myotubes, while reduced expression of the two proteins was observed following S-Rg3 treatment (Fig. 2B-D). The effect of S-Rg3 on apoptosis caused by DEX was also tested. The expression levels of apoptosis-related proteins, such as cleaved caspase-3, Bcl-2 
A

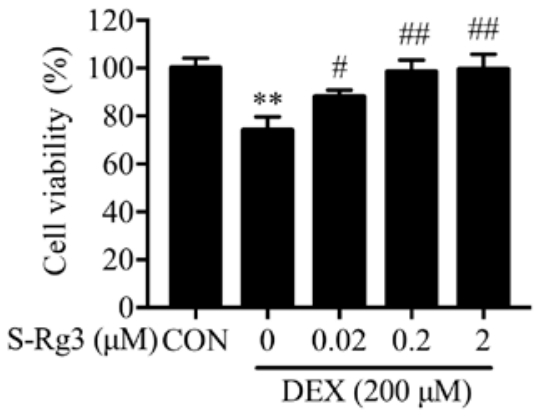

C

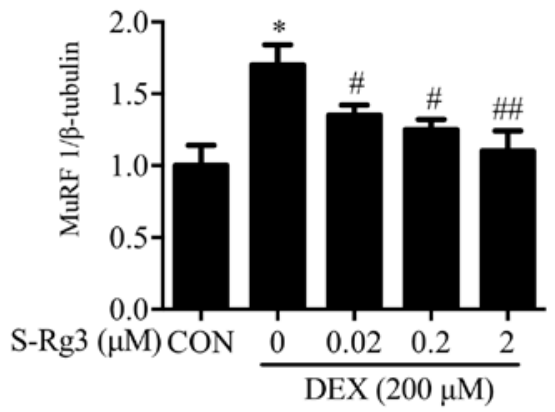

E

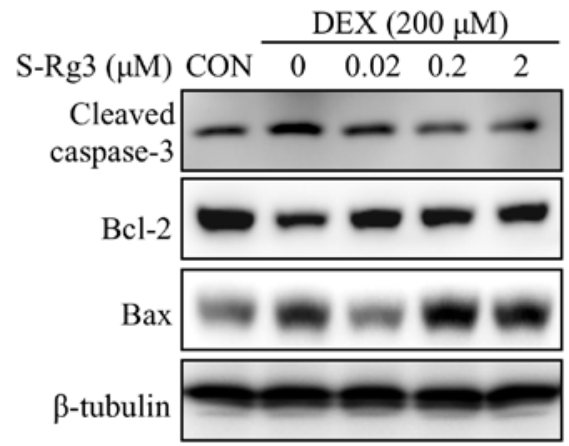

B

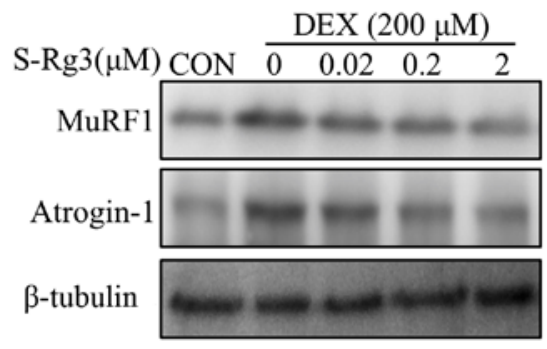

D

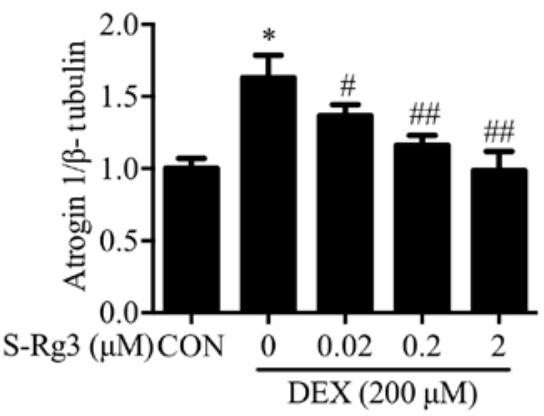

F

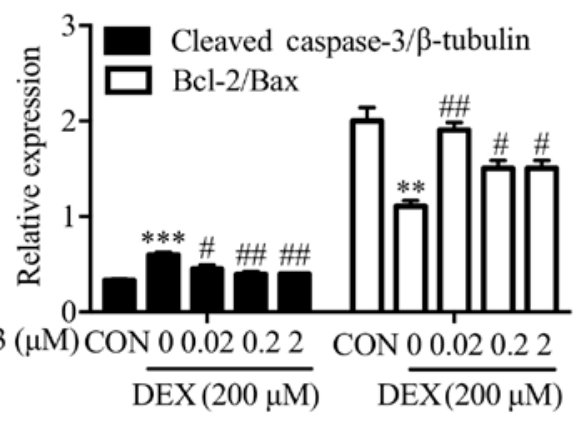

Figure 2. S-Rg3 reversal of DEX-induced C2C12 myotube atrophy. (A) After 24-h treatment of C2C12 myotubes with S-Rg3 (0.02, 0.2 and $2 \mu \mathrm{M})$ and DEX, MTT assays were conducted to measure viability of $\mathrm{C} 2 \mathrm{C} 12$ myotube cells. ${ }^{* *} \mathrm{P}<0.01$ vs. CON; ${ }^{~} \mathrm{P}<0.01$ and ${ }^{\# \#} \mathrm{P}<0.01$ vs. DEX. Results were analyzed via one-way ANOVA, with data expressed as the mean \pm standard deviation $(n=3)$. (B) To further analyze S-Rg3 treatment effects on skeletal muscle cell atrophy, atrogin-1 and MuRF1 expression levels were assessed via western blot analysis. (C and D) Relative atrogin-1 and MuRF1 expression levels were quantified via densitometric analysis, with $\beta$-tubulin serving as loading control. ${ }^{*} \mathrm{P}<0.05$ vs. $\mathrm{CON}$; ${ }^{*} \mathrm{P}<0.05$ and ${ }^{\# \#} \mathrm{P}<0.01$ vs. DEX. Results were analyzed via one-way ANOVA, with data expressed as the mean \pm standard deviation (n=3). (E) Following treatment of C2C12 myotubes with S-Rg3 and/or DEX for $24 \mathrm{~h}$, cleaved caspase-3, Bcl-2 and Bax levels were measured via western blot analysis. (F) Relative expression levels of cleaved caspase-3 and Bcl-2/Bax were semi-quantified by densitometric analyses based on $\beta$-tubulin as loading control. Results were analyzed using a one-way ANOVA. Data are shown as the mean \pm standard deviation $(\mathrm{n}=3) .{ }^{* *} \mathrm{P}<0.01,{ }^{* * *} \mathrm{P}<0.001$ vs. CON; ${ }^{\# \mathrm{P}}<0.05,{ }^{\# \#} \mathrm{P}<0.01$ vs. DEX. S-Rg3, 20(S)-ginsenoside Rg3; DEX, dexamethasone; MuRF1, muscle RING finger-1; atrogin-1, muscle atrophy F-box protein; CON, control group.

and Bax were examined by western blot analysis. The expression of cleaved caspase-3, which involved in the activation cascade of caspases responsible for apoptosis execution was decreased by $\mathrm{S}-\mathrm{Rg} 3$ treatment for $24 \mathrm{~h}$ in DEX-induced $\mathrm{C} 2 \mathrm{C} 12$ myotubes, and the ratio of the antiapoptotic protein $\mathrm{Bcl}-2$ and proapoptotic protein Bax was increased following treatment with S-Rg3 for $24 \mathrm{~h}$ (Fig. 2E-F).

Mitochondrial morphological changes induced by DEX are reversed by $\mathrm{S}-\mathrm{Rg} 3$ treatment. Previous studies conducted using a $\mathrm{C} 2 \mathrm{C} 12$ skeletal muscle cell line have demonstrated that mitochondrial morphological changes occur during mitophagy, a necessary cellular process for removing damaged mitochondria $(35,36)$. Using mitochondrial volume as a morphological indicator, DEX-induced mitochondrial injury was analyzed in $\mathrm{C} 2 \mathrm{C} 12$ myotubes using electron microscopy (Fig. 3A). Following DEX treatment, relatively large mitochondrial structural changes were observed, including swollen and broken mitochondria or the absence of mitochondria. These changes were ameliorated by treatment with $\mathrm{S}-\mathrm{Rg} 3$ as evidence that $\mathrm{S}-\mathrm{Rg} 3$ restored mitochondrial function (Fig. 3B and C).

DEX-induced mitochondrial dysfunction is alleviated by $S$-Rg3 treatment. To reveal whether an association exists between DEX-induced muscle atrophy and mitochondrial dysfunction, intracellular ATP levels and OCRs were 
A

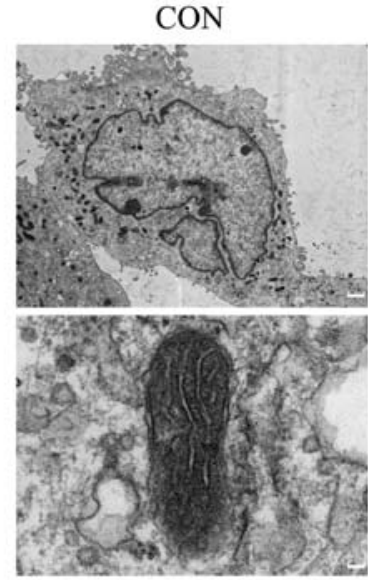

B

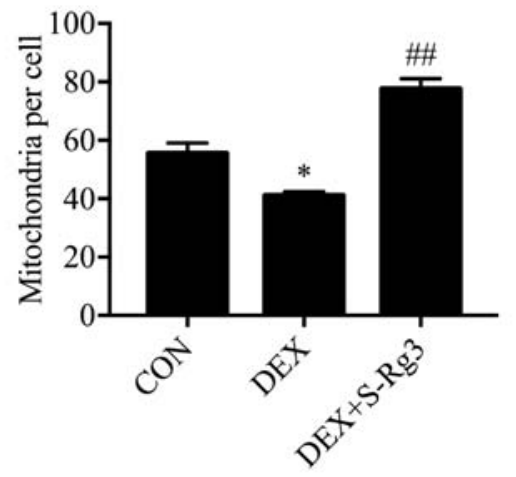

$\operatorname{DEX}(50 \mu \mathrm{M})$

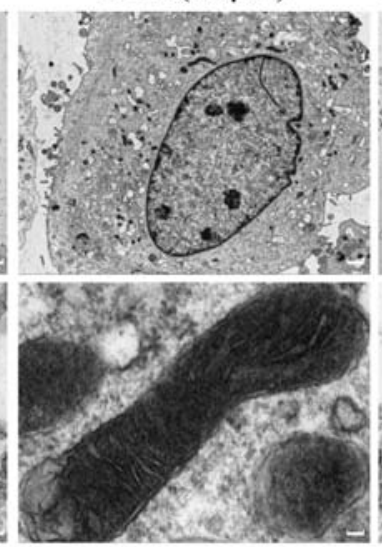

C

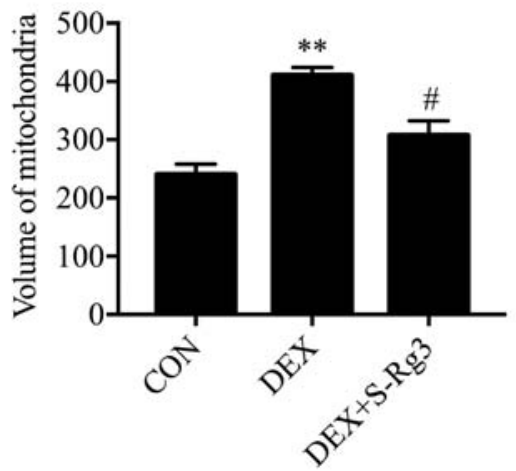

Figure 3. Reversal of DEX-induced mitochondrial morphological changes by S-Rg3 treatment. (A) Numerous mitochondria exhibited swelling and increased volume following $50 \mu \mathrm{M}$ DEX treatment. Scale bar, $5 \mu \mathrm{m}$ (top panels); scale bar, $200 \mathrm{~nm}$ (lower panels). (B) Mitochondrial numbers quantified using electron microscopic observations for each group. ${ }^{*} \mathrm{P}<0.05$ vs. CON; ${ }^{\#} \mathrm{P}<0.01$ vs. DEX. Results were analyzed via one-way ANOVA, with data expressed as the mean \pm standard deviation $(\mathrm{n}=3)$. (C) Mitochondrial volume as investigated by electron microscopic observations for each group. ${ }^{* *} \mathrm{P}<0.01$ vs. $\mathrm{CON}$; ${ }^{\#} \mathrm{P}<0.05$ vs. the DEX group. Results were analyzed via one-way ANOVA, with data expressed as the mean \pm standard deviation ( $=3$ ). DEX, dexamethasone; S-Rg3, 20(S)-ginsenoside Rg3; CON, control group.

measured in DEX-injured C2C12 myotubes. Ultimately, low ATP levels were observed following treatment with DEX that were increased following subsequent S-Rg3 treatment (Fig. 4A). In addition, S-Rg3 treatment reversed DEX-induced mitochondrial respiratory dysfunction (Fig. 4B), reversed DEX-induced reduction of mitochondrial membrane potential (an early sign of apoptosis in $\mathrm{C} 2 \mathrm{C} 12$ myotubes) (Fig. 4C) and reversed DEX-induced reductions of JC-1 aggregate levels from below control levels (as revealed via staining followed by flow cytometry) to levels above levels of untreated DEX-injured cells (Fig. 4D). Finally, as an additional assessment of whether mitochondrial dysfunction was induced by DEX, expression levels of key mitochondrial respiratory electron transport chain subunit proteins of complexes II, III and V were measured and significantly reduced expression levels of all key subunit proteins following DEX treatment were observed; these levels were increased following addition of S-Rg3 (Fig. 4E and F).

AMPK/FoxO3 signaling pathway inhibition by $\mathrm{S}-\mathrm{Rg} 3$ in the DEX-injured C2C12 myotube-based muscle atrophy model. A critical cellular energy sensor, AMPK (37), is regulated by the intracellular AMP to ATP ratio, with robust activation of AMPK induced by ATP deprivation (38). In the present study, AMPK phosphorylation increased markedly following 6-h
DEX treatment (Fig. 5A and B). In addition, increased nuclear and decreased cytoplasmic levels of transcription factor protein FoxO3, a known master regulator of muscle atrophy-associated E3 ligases MuRF1 and atrogin-1 (39), were observed. These changes were reversed by $\mathrm{S}-\mathrm{Rg} 3$ treatment, which decreased AMPK phosphorylation (Fig. 5A and B) and reduced FoxO3 nuclear translocation (Fig. 5C and D). Therefore, these data suggested that the AMPK/FoxO3 pathway is involved in the protective effect of $\mathrm{S}-\mathrm{Rg} 3$ on mitochondrial dysfunction.

\section{Discussion}

In spite of their beneficial anti-inflammatory and other clinically useful properties, GCs are known for numerous adverse myopathic effects (40). For example, high-dose synthetic GC DEX treatment has been observed to increase degradation and decrease synthesis of muscle protein that together led to reduced muscle mass and muscle fiber thinning (41). These myopathic effects appear to involve triggering of catabolic signals, including ubiquitin E3 ligases atrogin-1 and MuRF1 (42). As demonstrated in previous studies, such effects may be counteracted by ginsenoside Rg1 (22) and ginseng protein (43), which are reported to possess anti-atrophy effects, as well as by ginsenoside $\mathrm{S}-\mathrm{Rg} 3$, which has been shown to prevent myotube atrophy induced by TNF- $\alpha$ (24). The present 
A

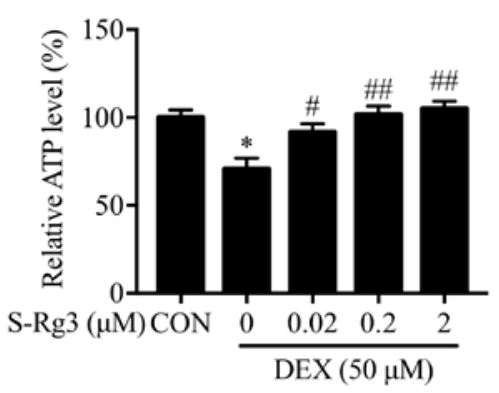

B

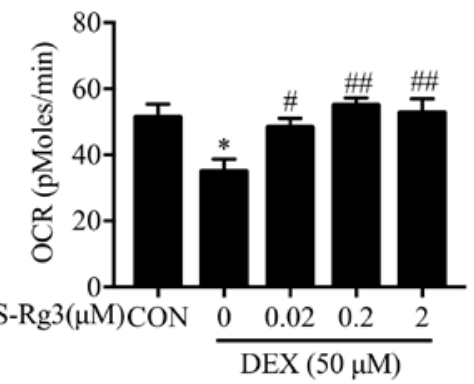

C

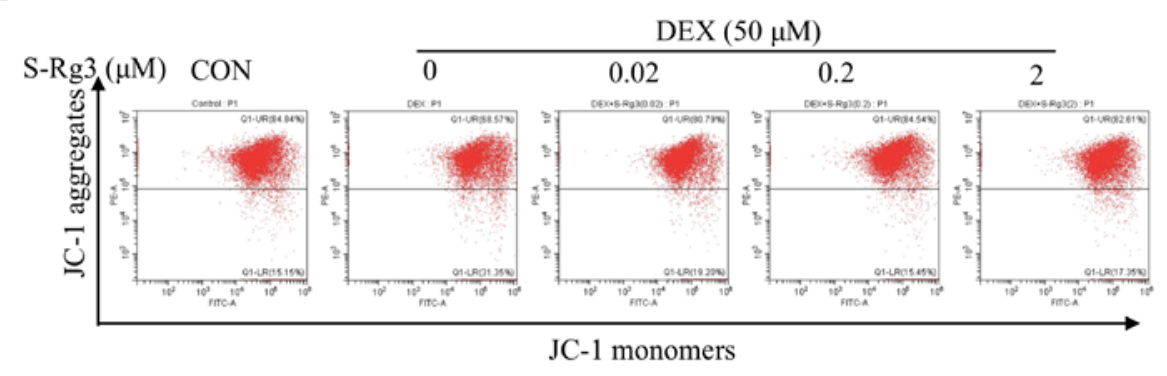

D

E
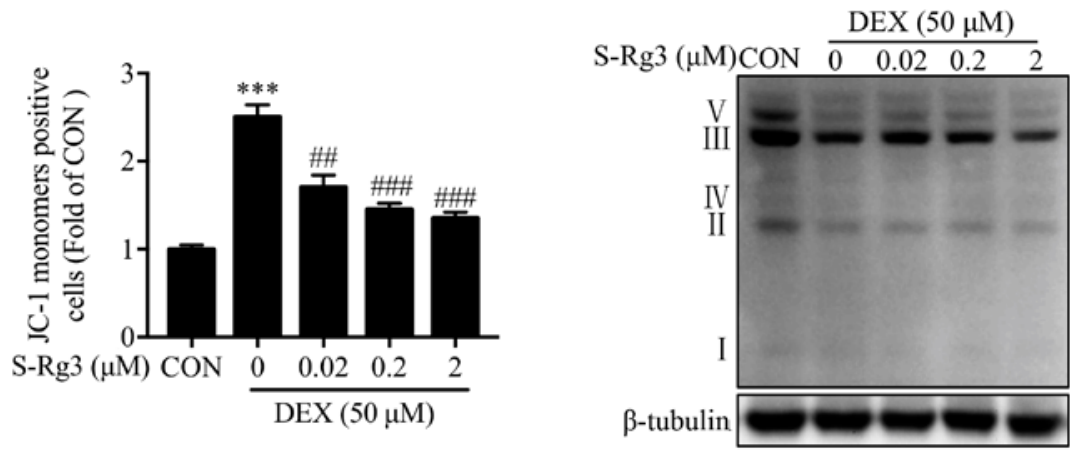

F

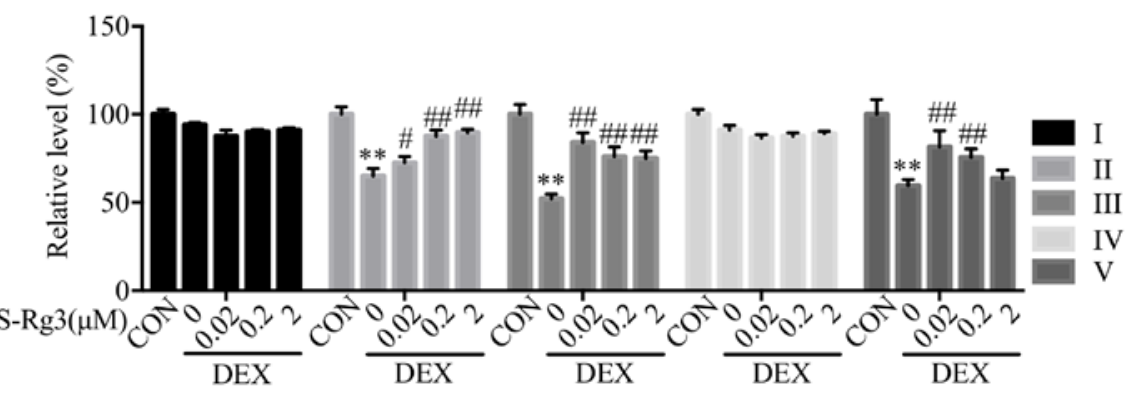

Figure 4. S-Rg3 reversal of mitochondrial dysfunction induced by DEX. (A) DEX-injured C2C12 myotube intracellular ATP level was increased by S-Rg3 treatment. ${ }^{*} \mathrm{P}<0.05$ vs. CON; ${ }^{\#} \mathrm{P}<0.05$ and ${ }^{\# \#} \mathrm{P}<0.01$ vs. DEX. Results were analyzed via one-way ANOVA, with data expressed as the mean \pm standard deviation $(\mathrm{n}=3)$. (B) OCR as measured by MitoXpress ${ }^{\circledR}$ Xtra Oxygen Consumption Assay measurement showing OCR decrease following 6-h DEX administration that was restored following administration of $\mathrm{S}-\mathrm{Rg} 3$. ${ }^{*} \mathrm{P}<0.05$ vs. CON; ${ }^{\text {"P }}<0.05$ and ${ }^{\# \#} \mathrm{P}<0.01$ vs. DEX. Results were analyzed via one-way ANOVA, with data expressed as the mean \pm standard deviation $(\mathrm{n}=3)$. (C) After 6-h DEX and/or S-Rg3 treatments, mitochondrial membrane potential was assessed following incubation of $\mathrm{C} 2 \mathrm{C} 12$ myotubes with the JC-1 probe followed by flow cytometric analysis. (D) Bar graph representing cells staining positive for JC-1 monomer then analyzed as in (C). ${ }^{* * *} \mathrm{P}<0.001$ vs. CON; ${ }^{\# \#} \mathrm{P}<0.01$ and ${ }^{\# \# \#} \mathrm{P}<0.001$ vs. DEX. Results were analyzed via one-way ANOVA, with data expressed as the mean \pm standard deviation $(n=3)$. (E) Representative western blots of DEX-injured C2C12 myotube complex I, II, III, IV and V proteins. (F) Relative expression of complex I, II, III, IV and V proteins quantified by densitometric analysis, with $\beta$-tubulin serving as loading control. Data are expressed as the mean \pm standard deviation $(\mathrm{n}=3)$. ${ }^{* *} \mathrm{P}<0.01$ vs. $\mathrm{CON} ;{ }^{\#} \mathrm{P}<0.05$ and ${ }^{\# \#} \mathrm{P}<0.01$ vs. DEX. Results were analyzed via one-way ANOVA, with data expressed as the mean \pm standard deviation $(\mathrm{n}=3$ ). S-Rg3, 20(S)-ginsenoside Rg3; DEX, dexamethasone; OCR, oxygen consumption rate; CON, control group.

study demonstrated that S-Rg3 prevention of myotube atrophy was linked to decreases in atrogin-1 and MuRF1 expression levels that subsequently corresponded to observed increase in viability of DEX-injured $\mathrm{C} 2 \mathrm{C} 12$ myotube cells. The present study also demonstrated that the in vitro experimental model of myotube atrophy employed could be used to evaluate drugs, such as S-Rg3, for effectiveness when used for treatment of muscle atrophy.

Muscle atrophy, a clinical disorder commonly associated with diabetes (44), chronic obstructive pulmonary disease (45) and other chronic diseases, negatively affects the quality of life of patients and promotes progression of pathological 
A

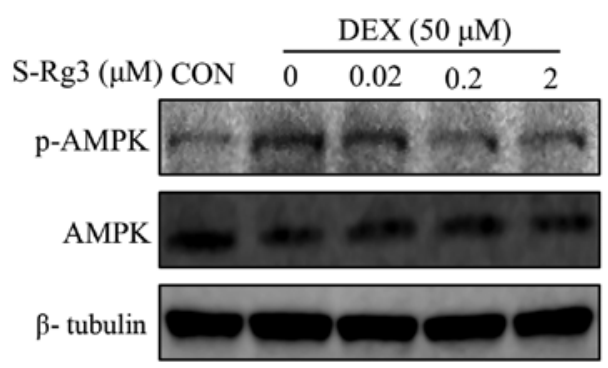

C
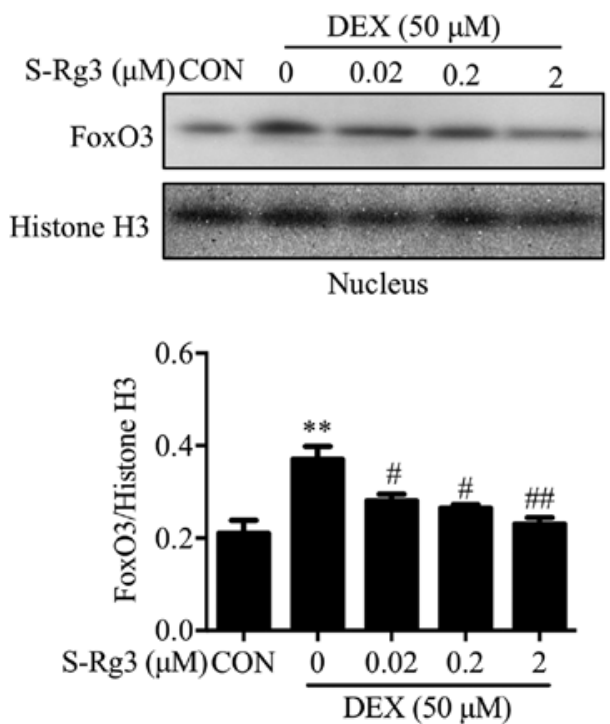

B

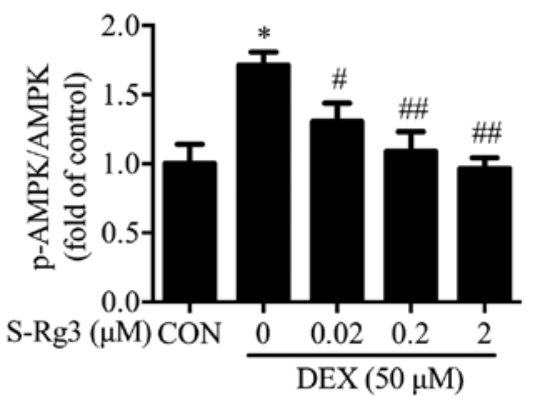

D
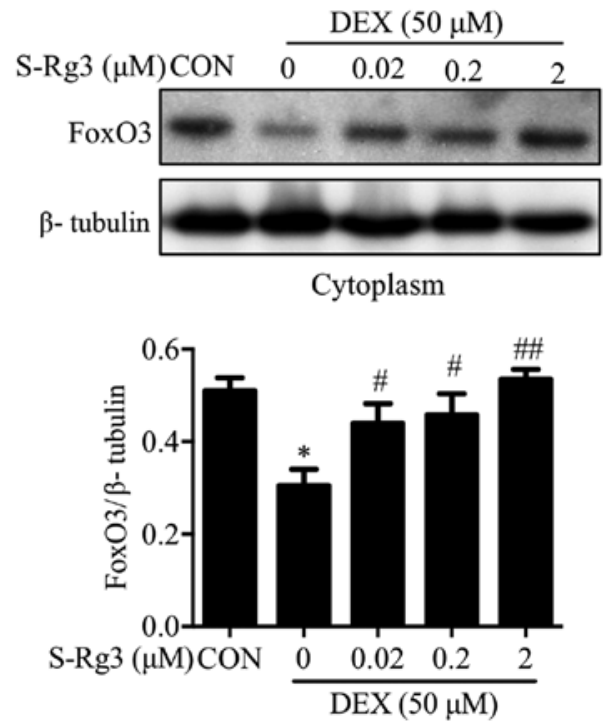

Figure 5. S-Rg3 inhibition of the AMPK/FOXO3 signaling pathway induced in C2C12 myotubes by DEX. (A) After 6-h S-Rg3 treatment, p-AMPK and AMPK levels in DEX-injured C2C12 myotubes were detected by western blot analysis, with $\beta$-tubulin serving as loading control. (B) Relative expression of p-AMPK/AMPK quantified using densitometric analysis, ${ }^{*} \mathrm{P}<0.05$ vs. CON; ${ }^{*} \mathrm{P}<0.05$ and ${ }^{\# \#} \mathrm{P}<0.01$ vs. DEX. Results were analyzed via one-way ANOVA, with data expressed as the mean \pm standard deviation $(\mathrm{n}=3)$. (C) To further explore $\mathrm{S}-\mathrm{Rg} 3$ effects, nuclear FoxO3 expression was studied via western blot analysis, with histone $\mathrm{H} 3$ serving as the nuclear protein loading control. Relative FoxO3 expression levels were semi-quantified via densitometric analysis. ${ }^{* *} \mathrm{P}<0.01$ vs. $\mathrm{CON}$; ${ }^{\#} \mathrm{P}<0.05,{ }^{\# \#} \mathrm{P}<0.01$ vs. DEX. Results were analyzed via one-way ANOVA, with data expressed as the mean \pm standard deviation ( $\mathrm{n}=3$ ). (D) To further explore S-Rg3 effects, cytoplasmic FoxO3 expression was studied via western blot analysis, with $\beta$-tubulin serving as the cytoplasmic protein loading control. Relative FoxO3 expression levels were semi-quantified via densitometric analysis. ${ }^{*} \mathrm{P}<0.05$ vs. CON; ${ }^{\#} \mathrm{P}<0.05$, ${ }^{\# \#} \mathrm{P}<0.01$ vs. DEX. Results were analyzed via one-way ANOVA, with data expressed as the mean \pm standard deviation (n=3). S-Rg3, 20(S)-ginsenoside Rg3; AMPK, AMP-activated protein kinase; FoxO3, forkhead box O3; p-, phosphorylated; DEX, dexamethasone; CON, control group.

disease (46). A growing body of evidence suggests that mitochondrial dysfunction is a key player in muscle atrophy caused by disuse and disease (47). Meanwhile, a previous study demonstrated that treatment with DEX can cause serious impairment of mitochondrial function manifesting as mitochondrial loss, dysfunctional mitochondrial respiration and disordered mitochondrial morphology and distribution (48). To counter such adverse DEX-induced effects, therapies targeting mitochondrial processes to increase mitochondrial biogenesis and/or enhance mitochondrial respiration are sought for prevention or treatment of muscle atrophy (49). In the present study, C2C12 myotube mitochondrial dysfunction induced by $50 \mu \mathrm{M}$ DEX administration was associated with decreased ATP level, mitochondrial respiration rate, mitochondrial membrane potential and mitochondrial complex II, III and V subunit protein levels. Notably, these pathological changes were prevented by $\mathrm{S}-\mathrm{Rg} 3$ treatment.

Mitochondria function as the main energy-producing organelles in cells, with mitochondrial functional disturbances leading to insufficient energy supply and activation of intracellular signaling pathways that culminates in AMPK activation, apoptosis and/or autophagy $(50,51)$. Notably, intracellular ATP deprivation triggers immediate activation of AMPK, an important energy sensor, that subsequently causes mitochondrial biogenesis (via PGC-1 $\alpha$ phosphorylation) or autophagy (via ULK1 phosphorylation) (52). As demonstrated in previous studies, AMPK participates in muscle atrophy in two ways, by phosphorylating FoxO3 and thereby directly controlling its nuclear translocation (53) and by participating in AMPK/FoxO3 pathway signaling triggered by mitochondrial fission (54). Our previous work demonstrated that S-Rg3 protects DEX-induced muscle atrophy probably by promoting AKT/mTOR phosphorylation and inhibiting FoxO3 nuclear transcription (25). The present study explored the role of mitochondrial function in muscle atrophy. The results showed that S-Rg3 treatment of DEX-injured myotubes decreased phosphorylation of AMPK and subsequently prevented nuclear translocation of FoxO3, effectively alleviating mitochondrial 
dysfunction and preventing muscle atrophy induced by DEX in vitro.

The present study studied GC-induced atrophy using an in vitro muscle myotube model to gain insights into underlying mechanisms for $\mathrm{S}-\mathrm{Rg} 3$ prevention of muscle atrophy. It was discovered that $\mathrm{S}-\mathrm{Rg} 3$ treatment restored mitochondrial function and promoted recovery from DEX-induced muscle atrophy by inhibiting the AMPK/FoxO3 pathway activated by DEX.

\section{Acknowledgements}

Not applicable.

\section{Funding}

The present study was supported by the National Natural Science Foundation of China (grant nos. U19A2013, U20A20402 and 81973813), the National Key Research and Development Program of China (grant no. 2017YFC1702106), the Administration of Traditional Chinese Medicine of Jilin Province (grant no. 2020171) and the Science and Technology Development Plan of Changchun City (grant no. 18YJ013).

\section{Availability of data and materials}

The data used and/or analyzed during the current study are available from the corresponding author on reasonable request.

\section{Authors' contributions}

MW analyzed data and wrote the manuscript. RJ and JL performed the experiments and conducted the analysis of data. XX and GS performed the statistical analysis of the data. DZ and LS designed the study and were involved in drafting the manuscript and confirm the authenticity of all the raw data. All authors read and approved the final manuscript.

\section{Ethics approval and consent to participate}

Not applicable.

\section{Patient consent for publication}

Not applicable.

\section{Competing interests}

The authors declare that they have no competing interests.

\section{References}

1. Ottens TH, Nijsten MW, Hofland J, Dieleman JM, Hoekstra M, van Dijk D and van der Maaten JM: Effect of high-dose dexamethasone on perioperative lactate levels and glucose control: A randomized controlled trial. Crit Care 19: 41, 2015.

2. Gong H, Liu L, Ni CX, Zhang Y, Su WJ, Lian YJ, Peng W, Zhang JP and Jiang CL: Dexamethasone rapidly inhibits glucose uptake via non-genomic mechanisms in contracting myotubes. Arch Biochem Biophys 603: 102-109, 2016.

3. Pasieka AM and Rafacho A: Impact of glucocorticoid excess on glucose tolerance: Clinical and preclinical evidence. Metabolites 6: 24, 2016.
4. Engel AG: Electron microscopic observations in thyrotoxic and corticosteroid-induced myopathies. Mayo Clin Proc 41: 785-796, 1966.

5. Oshima Y, Kuroda Y, Kunishige M, Matsumoto T and Mitsui T: Oxidative stress-associated mitochondrial dysfunction in corticosteroid-treated muscle cells. Muscle Nerve 30: 49-54, 2004.

6. Pereira RM and Freire de Carvalho J: Glucocorticoid-induced myopathy. Joint Bone Spine 78: 41-44, 2011.

7. Qin W, Pan J, Wu Y, Bauman WA and Cardozo C: Protection against dexamethasone-induced muscle atrophy is related to modulation by testosterone of FOXO1 and PGC-1 $\alpha$. Biochem Biophys Res Commun 403: 473-478, 2010.

8. Kuo T, Harris CA and Wang JC: Metabolic functions of glucocorticoid receptor in skeletal muscle. Mol Cell Endocrinol 380: 79-88, 2013.

9. Ochi A, Abe T, Nakao R, Yamamoto Y, Kitahata K, Takagi M, Hirasaka K, Ohno A, Teshima-Kondo S, Taesik G, et al: $\mathrm{N}$-myristoylated ubiquitin ligase Cbl-b inhibitor prevents on glucocorticoid-induced atrophy in mouse skeletal muscle. Arch Biochem Biophys 570: 23-31, 2015.

10. Jackman RW and Kandarian SC: The molecular basis of skeletal muscle atrophy. Am J Physiol Cell Physiol 287: C834-C843, 2004.

11. Gonnella P, Alamdari N, Tizio S, Aversa Z, Petkova V and Hasselgren PO: C/EBP $\beta$ regulates dexamethasone-induced muscle cell atrophy and expression of atrogin-1 and MuRF1. J Cell Biochem 112: 1737-1748, 2011.

12. Shen S, Liao Q, Liu J, Pan R, Lee SM and Lin L: Myricanol rescues dexamethasone-induced muscle dysfunction via a sirtuin 1-dependent mechanism. J Cachexia Sarcopenia Muscle 10: 429-444, 2019.

13. Weng J, Wang YH, Li M, Zhang DY and Jiang BG: GSK3 $\beta$ inhibitor promotes myelination and mitigates muscle atrophy after peripheral nerve injury. Neural Regen Res 13: 324-330, 2018.

14. Yoshioka Y, Kubota Y, Samukawa Y, Yamashita Y and Ashida H: Glabridin inhibits dexamethasone-induced muscle atrophy. Arch Biochem Biophys 664: 157-166, 2019.

15. Zhang SF, Zhang Y, Li B and Chen N: Physical inactivity induces the atrophy of skeletal muscle of rats through activating AMPK/FoxO3 signal pathway. Eur Rev Med Pharmacol Sci 22: 199-209, 2018

16. Hu R, Wang MQ, Liu LY, You HY, Wu XH, Liu YY, Wang YJ, Lu L, Xiao W and Wei LB: Calycosin inhibited autophagy and oxidative stress in chronic kidney disease skeletal muscle atrophy by regulating AMPK/SKP2/CARM1 signalling pathway. J Cell Mol Med 24: 11084-11099, 2020.

17. Paggio A, Checchetto V, Campo A, Menabò R, Di Marco G, Di Lisa F, Szabo I, Rizzuto R and De Stefani D: Identification of an ATP-sensitive potassium channel in mitochondria. Nature 572: 609-613, 2019.

18. Seok YM, Yoo JM, Nam Y, Kim J, Kim JS, Son JH and Kim HJ: Mountain ginseng inhibits skeletal muscle atrophy by decreasing muscle RING finger protein-1 and atrogin1 through forkhead box O3 in L6 myotubes. J Ethnopharmacol 270: 113557, 2020.

19. Lee MK, Choi JW, Choi YH and Nam TJ: Pyropia yezoensis protein prevents dexamethasone-induced myotube atrophy in C2C12 myotubes. Mar Drugs 16: 497, 2018.

20. Ma YL, Sun YZ and Yang HH: Protective effect of RenShen compound and DanHuang compound on muscle atrophy in suspended rats. Space Med Med Eng (Beijing) 12: 281-283, 1999 (In Chinese).

21. Takamura Y, Nomura M, Uchiyama A and Fujita S: Effects of aerobic exercise combined with panaxatriol derived from ginseng on insulin resistance and skeletal muscle mass in type 2 diabetic mice. J Nutr Sci Vitaminol (Tokyo) 63: 339-348, 2017.

22. Li F, Li X, Peng X, Sun L, Jia S, Wang P, Ma S, Zhao H, Yu Q and Huo H: Ginsenoside Rg1 prevents starvation-induced muscle protein degradation via regulation of $\mathrm{AKT} / \mathrm{mTOR} / \mathrm{FoxO}$ signaling in C2C12 myotubes. Exp Ther Med 14: 1241-1247, 2017.

23. Go GY, Lee SJ, Jo A, Lee J, Seo DW, Kang JS, Kim SK, Kim SN, Kim YK and Bae GU: Ginsenoside Rg1 from Panax ginseng enhances myoblast differentiation and myotube growth. J Ginseng Res 41: 608-614, 2017.

24. Lee SJ, Bae JH, Lee H, Lee H, Park J, Kang JS and Bae GU: Ginsenoside $\operatorname{Rg} 3$ upregulates myotube formation and mitochondrial function, thereby protecting myotube atrophy induced by tumor necrosis factor-alpha. J Ethnopharmacol 242: 112054, 2019. 
25. Wang M, Ren J, Chen X, Liu J, Xu X, Li X, Zhao D and Sun L: 20(S)-ginsenoside Rg3 promotes myoblast differentiation and protects against myotube atrophy via regulation of the Akt/mTOR/FoxO3 pathway. Biochem Pharmacol 180: 114145, 2020.

26. Rovetta F, Stacchiotti A,Faggi F, Catalani S, Apostoli P,Fanzani A and Aleo MF: Cobalt triggers necrotic cell death and atrophy in skeletal C2C12 myotubes. Toxicol Appl Pharmacol 271: 196-205, 2013.

27. Wang M, Chen X, Jin W, Xu X, Li X and Sun L: Ginsenoside Rb3 exerts protective properties against cigarette smoke extract-induced cell injury by inhibiting the p38 MAPK/NF- $\mathrm{KB}$ and TGF- $\beta 1 /$ VEGF pathways in fibroblasts and epithelial cells. Biomed Pharmacother 108: 1751-1758, 2018.

28. Meng F, Su X, Li W and Zheng Y: Ginsenoside Rb3 strengthens the hypoglycemic effect through AMPK for inhibition of hepatic gluconeogenesis. Exp Ther Med 13: 2551-2557, 2017.

29. Gwag T, Park K, Kim E, Son C, Park J, Nikawa T and Choi I: Inhibition of $\mathrm{C} 2 \mathrm{C} 12$ myotube atrophy by a novel HSP70 inducer celastrol, via activation of Akt1 and ERK1/2 pathways. Arch Biochem Biophys 537: 21-30, 2013.

30. Liu J, Peng Y, Wang X, Fan Y, Qin C, Shi L, Tang Y, Cao K, Li H, Long J and Liu J: Mitochondrial dysfunction launches dexamethasone-induced skeletal muscle atrophy via AMPK/FOXO3 signaling. Mol Pharm 13: 73-84, 2016.

31. Will Y and Dykens J: Mitochondrial toxicity assessment in industry-a decade of technology development and insight. Expert Opin Drug Metab Toxicol 10: 1061-1067, 2014.

32. Chen WC, Hsieh SR, Chiu CH, Hsu BD and Liou YM: Molecular identification for epigallocatechin-3-gallate-mediated antioxidant intervention on the $\mathrm{H} 2 \mathrm{O} 2$-induced oxidative stress in $\mathrm{H} 9 \mathrm{c} 2$ rat cardiomyoblasts. J Biomed Sci 21: 56, 2014.

33. Livak KJ and Schmittgen TD: Analysis of relative gene expression data using real-time quantitative PCR and the 2(-Delta Delta C(T)) method. Methods 25: 402-408, 2001

34. Huang Q, Lou T, Wang M, Xue L, Lu J, Zhang H, Zhang Z, Wang $\mathrm{H}$, Jing $\mathrm{C}$, Zhao $\mathrm{D}$, et al: Compound $\mathrm{K}$ inhibits autophagy-mediated apoptosis induced by oxygen and glucose deprivation/reperfusion via regulating AMPK-mTOR pathway in neurons. Life Sci 254: 117793, 2020

35. Troncoso R, Paredes F, Parra V, Gatica D, Vásquez-Trincado C, Quiroga C, Bravo-Sagua R, López-Crisosto C, Rodriguez AE, Oyarzún AP, et al: Dexamethasone-induced autophagy mediates muscle atrophy through mitochondrial clearance. Cell Cycle 13 : 2281-2295, 2014.

36. Huang Y, Chen K, Ren Q, Yi L, Zhu J, Zhang Q and Mi M: Dihydromyricetin attenuates dexamethasone-induced muscle atrophy by improving mitochondrial function via the PGC-1a pathway. Cell Physiol Biochem 49: 758-779, 2018.

37. Hardie DG, Ross FA and Hawley SA: AMPK: A nutrient and energy sensor that maintains energy homeostasis. Nat Rev Mol Cell Biol 13: 251-262, 2012.

38. Zheng WL, Wang BJ, Wang L, Shan YP, Zou H, Song RL, Wang T, Gu JH, Yuan Y, Liu XZ, et al: ROS-mediated cell cycle arrest and apoptosis induced by zearalenone in mouse sertoli cells via ER stress and the ATP/AMPK pathway. Toxins (Basel) 10: 24, 2018

39. Sandri M, Sandri C, Gilbert A, Skurk C, Calabria E, Picard A Walsh K, Schiaffino S, Lecker SH and Goldberg AL: Foxo transcription factors induce the atrophy-related ubiquitin ligase atrogin-1 and cause skeletal muscle atrophy. Cell 117: 399-412, 2004.

40. DiMauro S, Garone C and Naini A: Metabolic myopathies. Curr Rheumatol Rep 12: 386-393, 2010.
41. Kim JW, Ku SK, Han MH, Kim KY, Kim SG, Kim GY, Hwang HJ, Kim BW, Kim CM and Choi YH: The administration of Fructus Schisandrae attenuates dexamethasone-induced muscle atrophy in mice. Int J Mol Med 36: 29-42, 2015.

42. Shimizu N, Yoshikawa N, Ito N, Maruyama T, Suzuki Y, Takeda S, Nakae J, Tagata Y, Nishitani S, Takehana K, et al: Crosstalk between glucocorticoid receptor and nutritional sensor mTOR in skeletal muscle. Cell Metab 13: 170-182, 2011.

43. Jiang R, Wang M, Shi L, Zhou J, Ma R, Feng K, Chen X, Xu X, $\mathrm{Li} \mathrm{X}, \mathrm{Li} \mathrm{T}$ and Sun L: Panax ginseng total protein facilitates recovery from dexamethasone-induced muscle atrophy through the activation of glucose consumption in $\mathrm{C} 2 \mathrm{C} 12$ myotubes. Biomed Res Int 2019: 3719643, 2019.

44. Xu D, Jiang Z, Sun Z, Wang L, Zhao G, Hassan HM, Fan S, Zhou W, Han S, Zhang L and Wang T: Mitochondrial dysfunction and inhibition of myoblast differentiation in mice with high-fat-diet-induced pre-diabetes. J Cell Physiol 234: 7510-7523, 2019.

45. Gosker HR, Engelen MP, van Mameren H, van Dijk PJ, van der Vusse GJ, Wouters EF and Schols AM: Muscle fiber type IIX atrophy is involved in the loss of fat-free mass in chronic obstructive pulmonary disease. Am J Clin Nutr 76: 113-119, 2002.

46. Atherton PJ, Greenhaff PL, Phillips SM, Bodine SC, Adams CM and Lang $\mathrm{CH}$ : Control of skeletal muscle atrophy in response to disuse: Clinical/preclinical contentions and fallacies of evidence. Am J Physiol Endocrinol Metab 311: E594-E604, 2016.

47. Romanello V and Sandri M: Mitochondrial biogenesis and fragmentation as regulators of protein degradation in striated muscles. J Mol Cell Cardiol 55: 64-72, 2013.

48. Chen LE, Silver WP, Seaber AV, Korompilias AV and Urbaniak JR: Effects of dexamethasone on the contractile function of reperfused skeletal muscle. Microsurgery 17: 313-320, 1996.

49. Liu J, Peng Y, Feng Z, Shi W, Qu L, Li Y, Liu J and Long J: Reloading functionally ameliorates disuse-induced muscle atrophy by reversing mitochondrial dysfunction, and similar benefits are gained by administering a combination of mitochondrial nutrients. Free Radic Biol Med 69: 116-128, 2014.

50. Liu J, Deng K, Pan M, Liu G, Wu J, Yang M, Huang D, Zhang W and Mai K: Dietary carbohydrates influence muscle texture of olive flounder Paralichthys olivaceus through impacting mitochondria function and metabolism of glycogen and protein. Sci Rep 10: 21811, 2020.

51. Jager S, Handschin C, St-Pierre J and Spiegelman BM: AMP-activated protein kinase (AMPK) action in skeletal muscle via direct phosphorylation of PGC-1alpha. Proc Natl Acad Sci USA 104: 12017-12022, 2007.

52. Prieto I, Alarcón CR, García-Gómez R, Berdún R, Urgel T, Portero M, Pamplona R, Martínez-Ruiz A, Ruiz-Sanz JI, Ruiz-Larrea MB, et al: Metabolic adaptations in spontaneously immortalized PGC-1 $\alpha$ knock-out mouse embryonic fibroblasts increase their oncogenic potential. Redox Biol 29: 101396, 2020.

53. Han DS, Yang WS and Kao TW: Dexamethasone treatment at the myoblast stage enhanced $\mathrm{C} 2 \mathrm{C} 12$ Myocyte differentiation. Int J Med Sci 14: 434-443, 2017.

54. Romanello V, Guadagnin E, Gomes L, Roder I, Sandri C, Petersen Y, Milan G, Masiero E, Del Piccolo P, Foretz M, et al: Mitochondrial fission and remodelling contributes to muscle atrophy. EMBO J 29: 1774-1785, 2010.

This work is licensed under a Creative Commons Attribution-NonCommercial-NoDerivatives 4.0 International (CC BY-NC-ND 4.0) License. 